\title{
Mikroelektronik aus Österreich - Grundlage für Österreichs Innovations- und Wettbewerbsfähigkeit
}

\author{
B. Deutschmann
}

Online publiziert am 13. Februar 2018

(C) The Author(s) 2018. Dieser Artikel ist auf Springerlink.com mit Open Access verfügbar

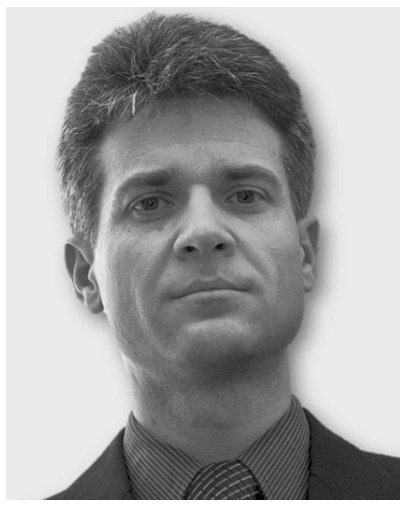

Univ.-Prof. Dipl.-Ing. Dr. Bernd Deutschmann
Liebe Leserinnen, liebe Leser,

wenn ein Industriezweig für sich in Anspruch nehmen darf, unsere Welt in den letzten Jahrzehnten nachhaltig verändert zu haben, dann ist es die Mikroelektronikindustrie. Es ist bemerkenswert, wie sich dieser Industriezweig seit der Erfindung des Mikrochips durch Jack S. Kilby vor knapp sechzig Jahren entwickelt hat.

Mikrochips können heutzutage Schaltungen mit vielen Milliarden elektronischer Bauelemente - insbesondere Transistoren - umfassen. Hochkomplexe Schaltungen wie Mikroprozessoren und Speicherchips sind auf wenigen Quadratmillimetern untergebracht und werden in modernsten Smart Factories zu einem großen Teil in Asien hergestellt.

Kaum jemand weiß aber, dass in so ziemlich jedem modernen Kraftfahrzeug, jedem Smartphone oder jedem elektronischen Reisepass Mikroelektronik verbaut ist, die hier in Österreich entwickelt und zum Teil auch gefertigt wird. In Österreich gibt es knapp 200 Unternehmen an ebenso vielen Standorten mit an die 63000 Personen, die im Bereich der Elektronikindustrie beschäftigt sind. Speziell im Großraum Graz existiert ein einzigartiger Cluster an Halbleiterunternehmen.

Aber wie kam es eigentlich dazu?

Begonnen hat alles Ende der 1970er Jahre, als sich die VOESTAlpine AG für eine mögliche Diversifizierung ihrer Produkt- und Dienstleistungspalette zu interessieren begann. Aufgrund der Erkenntnis, dass sich die Aktivitäten im Bereich der Stahlindustrie des Berg- und Maschinenbaus in den kommenden Jahren immer schwieriger gestalten würden, sah man einen Einstieg in die Elektronikindustrie, allen voran in die Produktion von integrierten Schaltungen, als eine Notwendigkeit, um an die internationalen Entwicklungen auf dem Gebiet der High-Tech-Elektronik anzuschließen; dies war ein wichtiger strategischer Beitrag zur Umstrukturierung der österreichischen Industrie.

Nach langer Suche nach einem Joint-Venture-Partner in den USA und in Japan gelang es, einen attraktiven Partner direkt aus dem Silicon Valley der USA nach Österreich zu holen: Die American Microsystems International, Inc., ein erfahrenes Unternehmen mit Stammsitz in Santa Clara, das bereits in den 1960er Jahren gegründet wor- den war und sich auf die Produktion von anwenderspezifischen integrierten Schaltkreisen (ASICs) spezialisiert hatte, war die Firma, mit der in Österreich der Grundstein für die Mikroelektronikindustrie gelegt wurde. Die Standortsuche für eine zukünftige Fertigungsstätte für integrierte Schaltungen fiel aufgrund von strukturpolitischen und sozialen Überlegungen auf die Steiermark - und zwar auf den Ort Unterpremstätten, genauer gesagt auf das Schloss Premstätten, welches als würdiger europäischer Hauptsitz der American Microsystems International, Inc. fungieren sollte.

Aus der Chronik der heute dort ansässigen ams AG wurde folgender Ausschnitt dazu entnommen:

"Da eine große Anzahl von wissenschaftlich ausgebildeten Fachkräften benötigt wird, kam grundsätzlich nur ein Standort in der Nähe einer Universität und mit breitem, qualifiziertem Arbeitskräfteangebot in Frage.

Mit der Errichtung dieses Werkes in der Umgebung von Graz ist außerdem die Schaffung eines, Silicon Valley-Effekts' beabsichtigt. Der Gründung dieses Elektronikwerkes soll die Ansiedelung weiterer Elektronikunternehmungen, so zum Beispiel Computerindustrie sowie mögliche Zulieferfirmen, in der näheren und weiteren Umgebung folgen."

Im originalen Silicon Valley in den USA ist aus einem einzigen Unternehmen, der Fairchild Semiconductor Corporation, seit den 1960er Jahren eine Flut von Spin-off-Unternehmen hervorgegangen, die durch eine beispiellose Reihe von technischen, geschäftlichen und kulturellen Innovationen das Silicon Valley zu einem weltweiten Zentrum unternehmerischer Aktivität und Technologieführerschaft machten. War es im Silicon Valley der Fairchild Semiconductor Corporation zu verdanken, so legte in Österreich sicherlich das JointVenture der VOEST-Alpine AG mit der American Microsystems International, Inc. das Fundament für einen führenden Industriestandort mit einer starken Position auf dem Weltmarkt.

Ähnlich wie im Silicon Valley gab es natürlich auch in Österreich einige Spin-offs, Zusammenschließungen und Neuansiedelungen, die zum explosiven unternehmerischen Wachstum der Region führten. Das "Silicon Valley Österreichs" beheimatet heute zahlreiche Unternehmen der Mikroelektronikindustrie. Global Player der Halbleiterhersteller wie Infineon Technologies AG, ams AG, NXP Semiconductors Austria $\mathrm{GmbH}$, Dialog Semiconductor $\mathrm{GmbH}$, STMicroelectronics Austria GmbH, CISC Semiconductor GmbH, Toshiba Electronics Europe GmbH, IDT Integrated Device Technology Inc, aber auch einige Start-ups in diesem Bereich wie die Panthronics AG, USound $\mathrm{GmbH}$ oder SteadySense $\mathrm{GmbH}$ haben sich seit dieser Zeit hier angesiedelt.

Deutschmann, Bernd, Institut für Elektronik, Technische Universität Graz, Inffeldgasse 12/I, 8010, Graz, Österreich (E-Mail: bernd.deutschmann@tugraz.at) 
Die Stärke des Standorts Österreich im Bereich der Mikroelektronik kommt aber nicht nur alleine aus dem Großraum Graz. Die Siemens AG gründete 1970 in Villach ein Bauelementewerk, um Glasdioden, später auch Fototransistoren zu fertigen. Schritt für Schritt wurden die Fertigungshallen für die Montage und den Test von ICS erweitert; seit den 1980iger Jahren von reinen Backend-Prozessen auch auf die IC-Fertigung. Heute betreibt die Infineon Technologies Austria AG dort ein Entwicklungszentrum und eine der weltweit modernsten Halbleiterfertigungsstätten.

Aber auch der Ballungsraum um Linz entwickelte sich zu einem wichtigen Elektronikstandort in Österreich. Hier wurde zum Beispiel vor vielen Jahren die Danube Integrated Circuit Engineering - DICE $\mathrm{GmbH} \&$ Co KG gegründet, die seit dem Jahr 2000 ein Unternehmen der Infineon Technologies AG ist.

Ein weiterer wichtiger Meilenstein in der österreichischen Halbleitergeschichte ist sicherlich auch die Ansiedelung des weltweit größten Halbleiterunternehmens Intel in Villach und Linz. Graz, Linz und Villach zählen inzwischen zu den wichtigsten Ballungsräumen der heimischen Elektronikindustrie.

Die technologische Stärke Österreichs liegt aber nicht nur darin, integrierte Schaltungen zu designen und zu fertigen, sondern diese auch gekonnt in Verbindung mit spezifischer Software in elektronische Geräte und Systeme einzubetten und damit elektronische Produkte erst intelligent zu machen. In diesem Bereich haben vor allem Electronic Based Systems (EBS) in den letzten Jahren stark an Bedeutung gewonnen. Sie beinhalten Elemente der Mikro- und Nanoelektronik, elektronische Komponenten, Module, Schnittstellen und Datenübertragung sowie eingebettete integrierte Systeme. Dadurch gelingt es, komplexe Aufgabenstellungen zu lösen. EBS sind nicht nur für eine Reihe von darauf aufbauenden elektronischen Produkten und Diensten unverzichtbar, sie bilden auch eine wichtige Grundlage für Innovationen speziell im Bereich des Internet der Dinge (IoT) und der Industrie 4.0.

Österreichs Stärke im Bereich der Elektronik liegt aber auch in der engen Verknüpfung der Industrie mit Forschungseinrichtungen, wie etwa der JOANNEUM RESEARCH Forschungsgesellschaft $\mathrm{mbH}$, der
CTR Carinthian Tech Research AG, der AIT Austrian Institute of Technology $\mathrm{GmbH}$, um nur einige zu nennen, sowie den zahlreichen Kooperationen der Industrie mit den heimischen Universitäten.

Aus der anwendungsorientierten Umsetzung der Forschungsergebnisse in der Industrie, in Verbindung mit den gut ausgebildeten Absolventinnen und Absolventen der technischen Schulen und Universitäten in Österreich sowie der guten Vernetzung im Rahmen zahlreicher nationaler Cluster, wie zum Beispiel Silicon Alps, ergibt sich ein einzigartiges Umfeld für die Entwicklung und Produktion neuer Technologien als Vorreiter, um Trends der Zukunft wie autonomes Fahren, Industrie 4.0 oder das Internet der Dinge hier in Österreich zu realisieren.

Die Mikroelektronikindustrie trägt seit vielen Jahren entscheidend dazu bei, die Innovationskraft in Österreich voranzutreiben. Grund genug, diesem Industriezweig einen eigenen Themenschwerpunkt der Zeitschrift e\&i zu widmen. Es freut mich besonders, dass es gelungen ist, Ihnen mit 13 Originalbeiträgen in diesem Schwerpunktheft einen Einblick in die Vielfalt der von Universitäten und Mikroelektronikunternehmen betriebenen Forschungsprojekte zu geben.

Ich möchte mich an dieser Stelle ganz herzlich bei den vielen $\mathrm{Au}$ toren der vorliegenden Beiträge sowie bei den Gutachtern der Originalarbeiten bedanken.

Ihnen, liebe Leserinnen und Leser, hoffe ich mit diesem Schwerpunktheft zum Thema Mikroelektronik aus Österreich einen informativen Überblick über eine der wichtigsten Schlüsseltechnologien für Innovationen in Österreich zu geben. Ich freue mich sehr, wenn es mir dadurch gelingt, die Begeisterung für Mikroelektronik auch nach außen zu tragen.

Ich wünsche Ihnen viel Vergnügen beim Lesen der Beiträge!

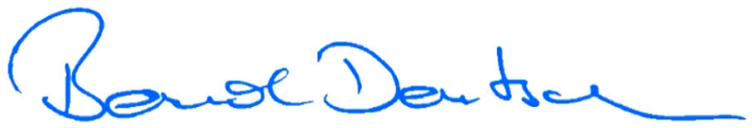

\section{Danksagung}

Open access funding provided by Graz University of Technology. 